\title{
A Method of Developing a Generic Social Network
}

\author{
Swapnil S. Ninawe and Pallapa Venkataram
}

\begin{abstract}
The rapid development of communication and networking has lessened geographical boundaries among actors in social networks. In social networks, actors often want admittance and permission over databases depending upon their rights, privacy, context, privileges, etc. Managing and handling knowledge based admittance of actors is complex and hard for which broad range of technologies need to be called. Admittance based on dynamic rights and circumstances of actors impose major tasks on systems. In this paper, we present a Generic Social Network (GSN) to render admittance to actors in social networks along with permission over databases taking privacy and status of them into consideration. The designed GSN is tested over an Agriculture Social Network (ASN) which utilises distinct rights and privileges of actors related to the agriculture occupation, and provides admittance to actors in it.
\end{abstract}

Index Terms-Actor, admittance, database, knowledge.

\section{INTRODUCTION}

A social network is a social structure between individuals also known as actors and organizations. Social network can also be defined as a group of actors connected by a set of relationships which are continuously changing. As an important research area, developing a social network focuses on relationships along with rights associated with those entities. Once the social network is constructed, it could be used to analyze knowledge discovery, like finding access, searching influential actors, groups, etc. In general developing a social network covers the area of any network, and metrics used are based on the mathematics of graph theory regardless of the connections. After constructing the social network by deciding upon its actors and relations, analysis is performed for knowledge discovery which is actually the ultimate goal.

With the advancement in the information technology, social networks [1]-[3] have influenced their actors of different regions to share the information. The main goal of social networks is to make the information space, where actors can share information like thoughts, personal data, events, etc. It shares the basic purpose of interaction and communication, and specifies goals and patterns that vary significantly across different permission [4]-[7] information. Structural variations [8], visibility of information [9] and admittance [10] are the significant characteristics of social networks. The most distinguishing features of social networks are relationships among social entities, patterns and deduction of these, while linking structure of social relationships to behaviors of actors participating in it. It renders actors to connect with other actors of similar interests

Manuscript received May 10, 2013; revised June 25, 2013.

The authors are with the Electrical Communication Engineering Department, Indian Institute of Science, India (e-mail: swapnil.ninawe@gmail.com, swapnil@ece.iisc.ernet.in, pallapa@ece.iisc.ernet.in). when physical barrier might have kept them apart. The popularity of social networks has increased because they enable researchers to study social network actors and relationships among them.

Admittance of actors based on knowledge has become important for people to have supportive and diversified social connections; some of the nature of the information might be context sensitive. In social networks, actors often want to acquire multiple resources, databases, etc., which may or may not be in the reach of their permission norms. The traditional mechanisms are built on few parameters such as actor's specified preferences, static denial policies, etc. It does not involve context [11], dynamic rights [12], privacy [13], trust [14], and other significant factors of actors that are needed to be taken into account, and requires dynamic updating of rights according to actors. The mechanisms that are currently deployed are unable to render the required admittance and permissions to actors. Innumerable number of actor uses and dynamic changing context imposes difficulties for auto updating various databases. Mechanisms [15]-[17] based on description of actor's profiles, preferences, privacy handling and social groups are difficult to address. Dynamic data variations, changing relations and non-availability of actors at all times imposes complexities for the admittance in social networks.

\section{A. Proposed Idea}

In this paper, we propose a Generic Social Network (GSN) which involves different information of actors like personal information, professional information, history, learning privileges, initial rights, etc. Depending upon multiple rights that are associated with each actor, permission over various databases is provided. Dynamic variation of rights along with privacy, relations and status of actors are the key factors in provision of admittance of actors and permission over databases in social networks.

\section{B. Organisation of the Paper}

The organisation of the rest of the paper is as follows. Section II covers some of the existing mechanisms used in social networks. Design of a generic social network and the proposed GSN is presented in Section III with an application of a social network for the agriculture in Section IV. Simulation environment and simulation results are covered in Section V and Section VI respectively, followed by conclusion in Section VII. The results obtained are quite encouraging for admittance of actors and permission over databases in social networks.

\section{SOME OF THE EXISTING MeChANISMS FOR SOCIAL NETWORKS}

Several research works exists on developing traditional network and not in social network, where a framework with 
the purpose of helping the development of social network in an ad hoc mobile network environment was presented [18], and provided support for the developer in the creation of interfaces. Block modeling and role analysis in multirelational networks was studied [19], and interpretation of various methods that presented actors, positions, roles and group concepts was carried out. Community evolution mining in dynamic social networks was implemented in [20], where the events detected by the framework was supplemented by extraction and investigation of the topics discovered for each community. Michele [21] showed graph evolution rules which helped in analyzing the evolution of large networks and could be used to predict the future creation of links among nodes, whereas adaptive algorithms for detecting community structure in dynamic social networks were implemented [22] and demonstrated bright applicability of algorithms.

Multi-level multi-theoretical model which gave a theoretical framework to explain the evolution of communication networks within teams was shown in [23], and enabled researchers to analyze dynamic network patterns of virtual teams. A novel model for social communities that captured their unique dynamic nature in terms of community structure and interest were presented [24] demonstrating the high degree of stability. Kyung Soo [25] suggested a noble method to grasp information of anonymous users through relationship information available and their psychology that was reflected on texts. An insight on how Malaysians share knowledge and benefits that they obtain through online social networking was shown in [26] and proved that social networking culture is related to knowledge sharing as a way of life. Traditional and novel social analysis tools were used [27] to study the emergence of interests around certain topics, the evolution of collaborations and identifying potential for better cooperation. Evolution of the social network of scientific collaborations were presented [28] and proposed a simple model that captured the network's time evolution.

\section{DESIGN OF A GENERIC SOCIAL NETWORK}

In this section we present a generic design of a simple social network with multiple actors and databases. Consider actors $a_{1}, a_{2}, \ldots, a_{k}$ relations from the set $\left\{r_{i 1}, r_{i 2}, \ldots, r_{i j}\right\}$, where $r_{i j}$ describes the relation between $a_{i}$ and $a_{j} . r_{i j}$ is the function of history, preferences and context between actors $a_{i}$ and $a_{j}$ Now each actor will try to link with another actor with help of the relations forming a social network. A collection of such social network will form an extended social network. For example, consider an actor $a_{i}$, who want to enter into a well established social network of actors $a_{1}, a_{2}, \ldots, a_{k}$. Admittance to the actor $a_{i}$ in the social network can be allowed based on the profile, preferences, history and context parameters of theactor $a_{i}$ with other actors. The intension is to add more and more suitable actors in the social network which will ultimately form the extended social network. Addition of new actors in the social network is significant for the development of the social network.

The objective is the study of social structures among actors, and to render coordination among them with their rights over databases $\left\{D_{1}, D_{2}, \ldots, D_{n}\right\}$ as shown in Fig. 1. Nomenclature used in the designed generic social network such as actor, groups, rights and databases are described in Table I.

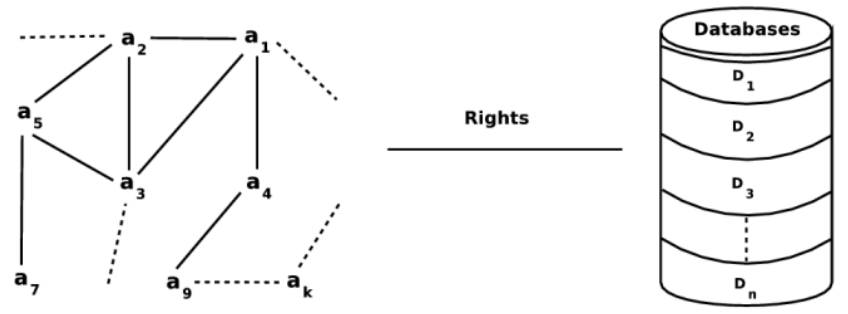

Fig. 1. The social network.

TABLE I: NOMENCLATURE USED IN THE SOCIAL NETWORK

\begin{tabular}{ll} 
Terms & Definition \\
\hline (1) Actor & An entity that can participate in social networks \\
(2) Group & A collection of actors \\
(3) Rights & Rights associated with actors in social networks \\
(4) Knowledge Base & A collection of knowledge databases \\
\hline \hline
\end{tabular}

\section{A. Proposed GSN}

In this subsection, we discuss the GSN which runs as supporting system to provide admittance to actors, and initially gathers information about actors such as Primary Rights (PR), Group Rights (GR) and Relation Rights (RR). Based on the circumstances and status of actors, the system provides permission to actors over databases in social networks.

Dynamic change of rights based on the circumstances of actors is the key factor in the proposed GSN. We discuss the architecture of the GSN (shown in Fig. 2) in detail each of the component and functionality. The main component of the system is the coordination module which coordinates with multiple modules, and functions of respected modules are as follows.

- Coordination module is responsible for coordinating with multiple modules. It takes various parameters from monitor module and provides it to control module, and supplies it to the update module.

- Monitor module is responsible for monitoring different rights such as PR, GR and RR. Each time the rights are updated, the monitor module continuously monitors these rights in real time.

- Control module creates logic for admittance of actors and permission over databases. The logic takes into account the dynamic variation of rights and calculates updated rights for a particular actor.

- Update module is responsible for updating multiple rights of actors.

Rights used in the GSN are:

1) Primary Rights (PR): Access rights associated with an actor over databases as individually. For example, consider an actor $a_{i}$ who is part of a social network and has some influence in it because of his credits.

2) Group Rights (GR): Access rights associated with an actor over databases in a particular group. For example, an actor belonging to a group has some different rights because he belongs to that particular group. 
3) Relation Rights (RR): Access rights associated with an actor with other actor over databases in different group. For example, consider an actor in relation with another actor who has influence in the social network, has different rights due to relations.

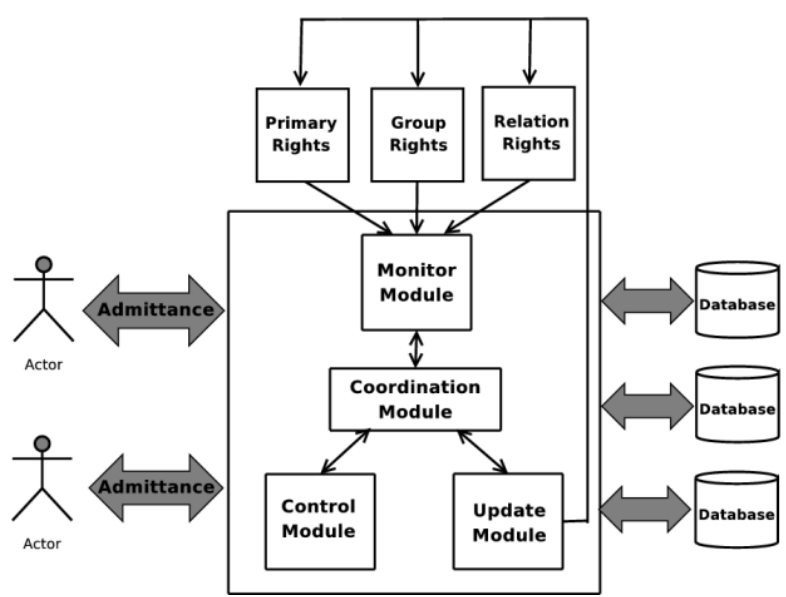

Fig. 2. The generic social network architecture.

The privacy of actors is taken into consideration based on the Actor Privacy Settings (APS), relation, status and the Permission Provider Privacy Settings (PPPS). Admittance based on PR, GR and RR utilises both APS and PPPS. For example, consider an actor $a_{i}$ want to communicate with an actor $a_{j}$, denoted as $\left(a_{i}:\right.$ info $\left.: a_{j}\right)$, the dynamic policies can be built depending on significant parameters of the actors $a_{i}$ and $a_{j}$ from the set $£=\{$ Group mate, Database access,

History, Resource access $\}$. If $\left(a_{i}:\right.$ info: $\left.: a_{j}\right)$ is greater than some threshold $(\chi), a_{i}$ can communicate with $a_{j}$, otherwise, communication is denied. Parameters used to compute the rights in the GSN are described in TABLE II along with the data structure for Actor in TABLE III.

\section{Design of the Agriculture BASEd Social Network SPECIFICATIONS USING THE GENERIC SOCIAL NETWORK}

In this section, we demonstrate the GSN functioning over the Agriculture Social Network (ASN) which is the combination of actors, relations, databases and rights. We have considered a typical 25 actors based ASN with five databases as shown in Fig. 3. Some of the terms associated with the ASN are described in TABLE IV. Nomenclature used in the ASN are:

1) Actor: Actors like scientist, banker, farmer and labourer.

2) Group: Actors belonging to the same profession.

3) Primary rights of Project Assistant: Rights associated with Project Assistant over database as individually.

4) Group rights of Project Assistant: Rights associated with Project Assistant over database in scientist group.

5) Relation rights of Project Assistant: Rights associated with Project Assistant over database corresponding to relations with other actors.

In the ASN, actors like scientist, banker, farmer and labourer with relationships such as strong, reasonable, fair or weak exists among them which are used for permission over databases like seed, soil, crop, etc. For example, a farmer might approach to a banker for loan; hence supplier-client fair relationship exists among them. Another strong relationship is hirer-worker which exists between a farmer and a labourer.

In the ASN, actors are divided into groups such as Scientist (S), Banker (B), Farmer (F) and Labour (L) with respective PR, GR and RR.

TABLE II: PARAMETERS CONSIDERED TO COMPUTE RIGHTS IN SOCIAL NETWORKS

\begin{tabular}{ll}
\hline \hline Terms & Parameters \\
\hline Profile $\left(p f_{a_{i}}\right)$ & Age, Gender, Education, etc. \\
Preference $\left(p r_{a_{i}}\right)$ & Data sharing, Data filter, etc. \\
Access Privilege $\left(a p_{a_{i}}\right)$ & Read, Write, New post, etc. \\
Context $\left(c_{a_{i}}\right)$ & Location, Time, Position, etc. \\
Interest Similarity $\left(i s_{a_{i}}\right)$ & Working on same project, data, etc. \\
Coordination $\left(c o_{a_{i}}\right)$ & Working together, Achieving goals, etc. \\
History $\left(h_{a_{i}}\right)$ & Previous contacts, interaction, etc. \\
Relation $\left(r_{a_{i}}\right)$ & Strong, Fair, Weak, etc. \\
\hline \hline
\end{tabular}

TABLE III: DATA STRUCTURE FOR ACTOR

\begin{tabular}{ccc}
\hline \multicolumn{2}{c}{ TABLE III: DATA STRUCTURE FOR ACTOR } \\
\hline $\begin{array}{ccc}\text { Actor Name } \\
\text { (AN) }\end{array}$ & Database (DB) & $\begin{array}{c}\% \text { ofmission of } a_{i} \text { over } \\
\text { databases }\left(P O P_{a_{i}}^{D_{x}}\right.\end{array}$ \\
\hline$a_{1}$ & $D_{3}$ & $P O P_{a_{1}}^{D_{3}}$ \\
$\cdot$ & $\cdot$ & $\cdot$ \\
$\cdot$ & $\cdot$ & $\cdot$ \\
$a_{n}$ & $D_{k}$ & $P O P_{a_{n}}^{D_{k}}$ \\
\hline \hline
\end{tabular}
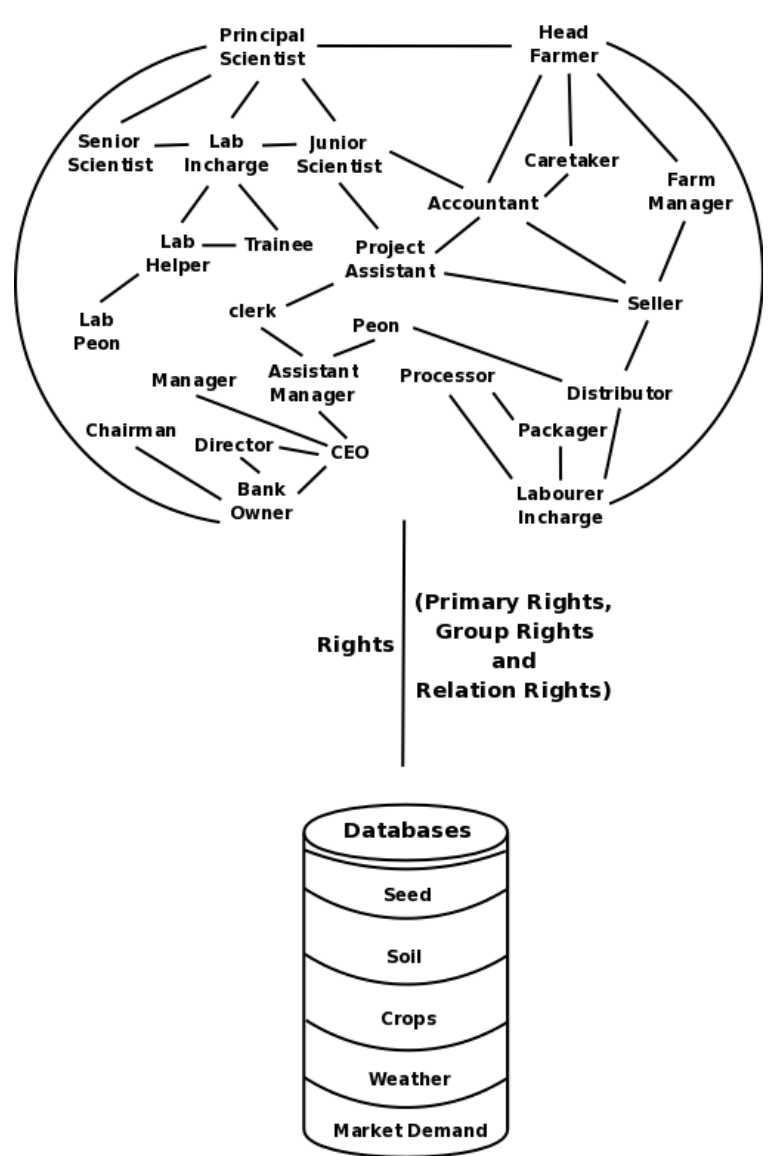

Fig. 3. A typical application for the agriculture social network. 
TABLE IV: NOMENCLATURE USED IN THE ASN

\begin{tabular}{|c|c|}
\hline Terms & Definition \\
\hline $\begin{array}{l}\text { (1) Actor } \\
\text { (2) Group }\end{array}$ & $\begin{array}{l}\text { scientist, banker, farmer, labour } \\
\text { Actors belonging to the same } \\
\text { profession }\end{array}$ \\
\hline $\begin{array}{l}\text { (3) Primary Rights of Project } \\
\text { Assistant (PA) over crop database } \\
\text { (4) Group Rights of Project } \\
\text { Assistant (PA) over crop database } \\
\text { (5) Relation Rights of Project } \\
\text { Assistant (PA) with Seller (SL) over } \\
\text { crop database } \\
\text { (6) Knowledge Base }\end{array}$ & $\begin{array}{l}\text { Rights associated with PA } \\
\text { over crop database as individual } \\
\text { Rights related with PA in the } \\
\text { scientist group over crop } \\
\text { database } \\
\text { Rights pertaining to PA with SL } \\
\text { over crop database }\end{array}$ \\
\hline
\end{tabular}

\section{SimUlation ENVIRONMENT}

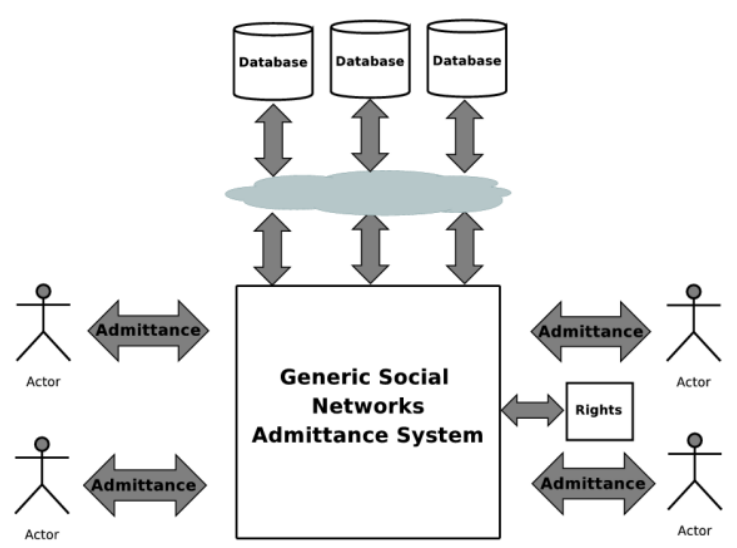

Fig. 4. Simulation environment.

We have allocated rights of actors based on the agriculture system, i.e., four groups of actors and five databases with hundred actors frequency. Initially all actors are assigned their respective PR, GR and RR. As actors arrive the system randomly, the GSN dynamically monitors different rights depending upon circumstances of actors, the admittance in social networks and permission over databases is rendered, and corresponding PR, GR and RR are updated as shown in Fig. 4.

\section{Simulation Results}

We have simulated the GSN (on Java platform) and result is shown in Fig. 5, where time taken for generating actors and their access over database is plotted, and shows that the time taken to access database is well below $8 \%$ after actors are being generated.

The graph (shown in Fig. 6) is plotted as variation of rights against admittance of actors. It shows variation of PR, GR and RR of actors taken over hundred admittance frequencies. The bar graph shown in Fig. 7 shows percentage of admittance based on PR, GR and RR against rights. It shows that admittance provided based on PR, GR and RR is $23 \%, 49 \%$ and $28 \%$ respectively.

Number of admittance of actors against time is plotted in Fig. 8 which shows number of actors admittance varied between 13 to 25 . Another graph in Fig. 9 describes the comparison between authorised admittance (87\%) and unauthorised admittance (13\%).

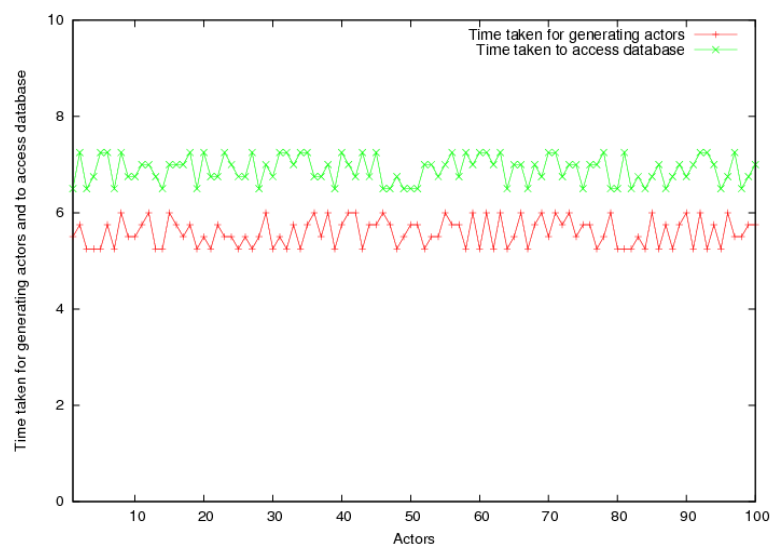

Fig. 5. Time taken for generating actors and to access database $v s$ actors.

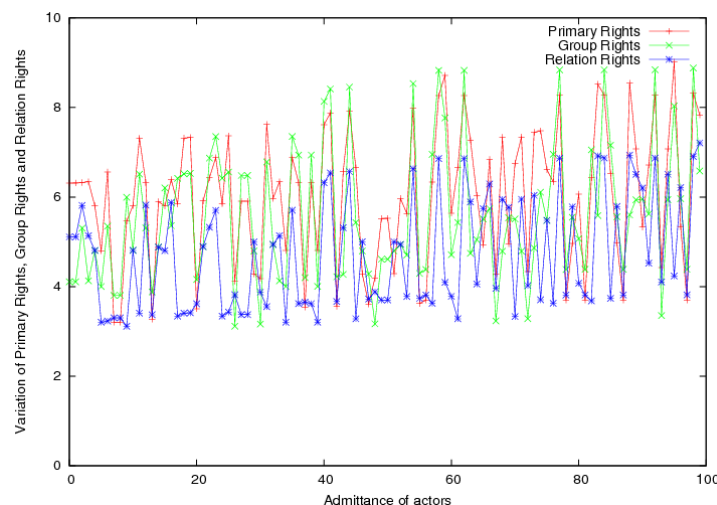

Fig. 6. Variation of primary rights, group rights and relation rights $v s$. admittance of actors.

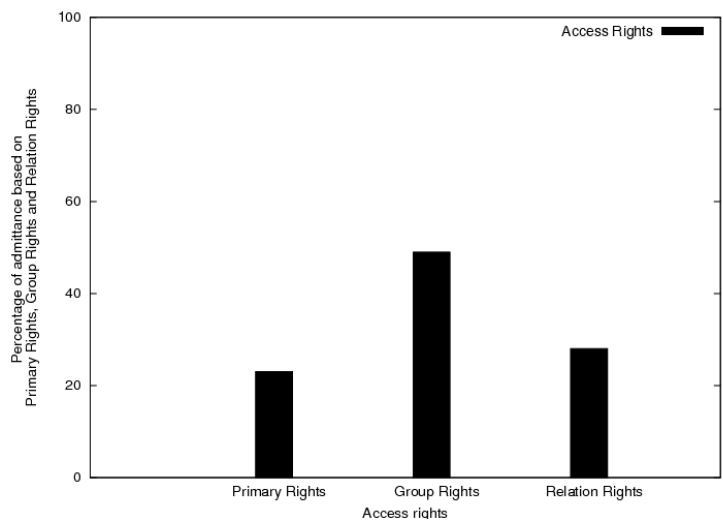

Fig. 7. Percentage of admittance based on primary rights, group rights and relation rights $v s$ rights.

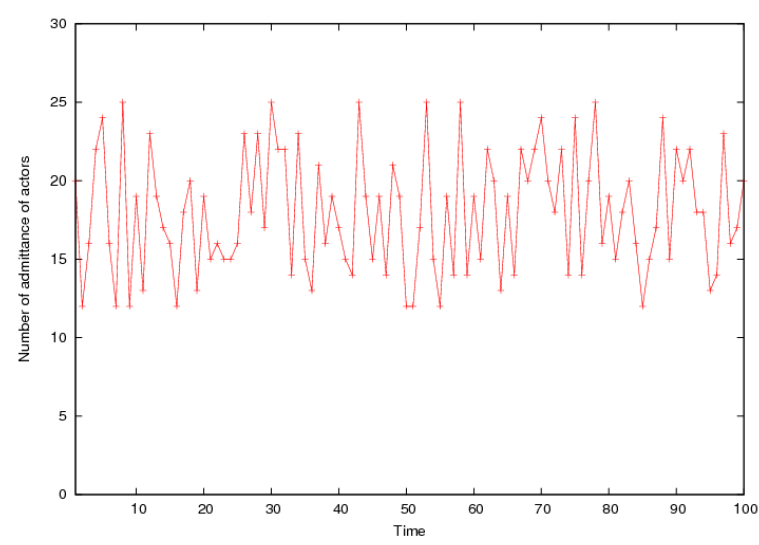

Fig. 8. Number of admittance of actors vs. time. 


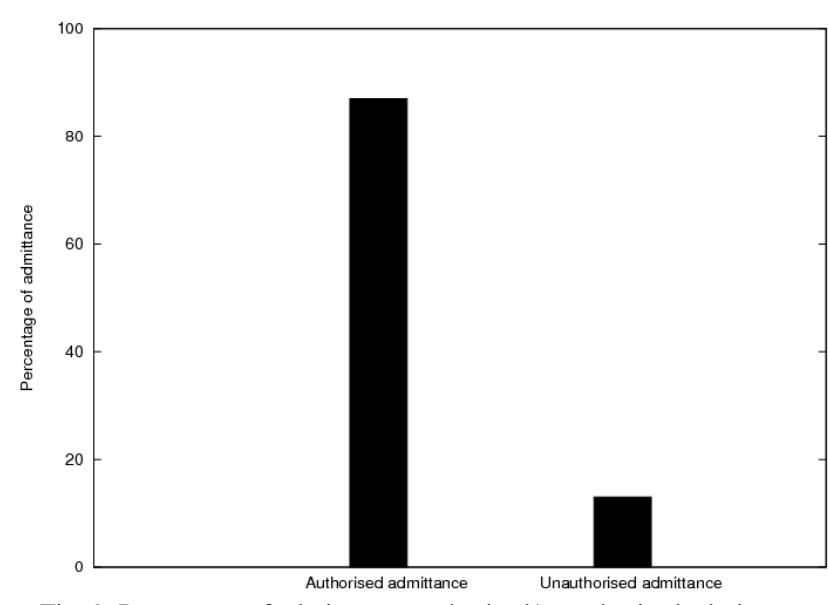

Fig. 9. Percentage of admittance: authorised/unauthorised admittance.

\section{CONCLUSION}

A method of developing a generic social network was presented, which facilitated admittance of actors and permission over databases in the social network based on their rights. It utilised various parameters of actors and dynamically computed multiple rights.

The GSN was designed for the agriculture social network, where the admittance of actors related to the agriculture occupation and permission over multiple databases was carried out. Rights of actors were dynamically updated, and permission over databases like seed, soil, crop, etc. was rendered.

Graphs obtained were consistent with the generalized formulation and the application. The proposed scheme can be easily deployed to obtain admittance of actors and permission over databases in social networks.

\section{REFERENCES}

[1] D. V. Greetham, R. Hurling, G. Osborne, and A. Linley, "Socia networks and positive and negative affect," Procedia- Social and Behavioral Sciences, vol. 22, pp. 4-13, 2011.

[2] P. Brdka, S. Saganowski, and P. Kazienko, "Ged: the method for group evolution discovery in social networks," Social Network Analysis and Mining, pp. 1-14, 2009.

[3] J.-T. Qiu, Z.-X. Lin, C.-J. Tang, and S.-J. Qiao, "Discovering organizational structure in dynamic social network," Data Mining, 2009. ICDM '09, Ninth IEEE International Conference on, pp. 932-937, Dec. 2009.

[4] E. Avram and I. Priescu, "Access to information and empowerment perspectives in health services," Procedia - Social and Behavioral Sciences, vol. 33, pp. 949-953, 2012.

[5] S. Furnell and R. A. Botha, "Social networks access all areas?" Compute Fraud and amp Security, vol. 2011, no. 5, pp. 14-19, 2011.

[6] S. Hiremagalore, C. Liang, A. Stavrou, and H. Rangwala, "Improving network response times using social information," Social Network Analysis and Mining, pp. 1-12, 2012.

[7] J.-G. Li, Y.Tang, C.-J. Mao, H.-J. Lai, and J. Zhu, "Role based access control for social network sites," Pervasive Computing (JCPC), 2009 Joint Conferences on, pp. 389 -394, Dec. 2009.

[8] Journal of Communications and Networks, vol. 13, no. 2, pp. 175-186, April 2011.

[9] B. Zhou and J. Pei, "Preserving privacy in social networks against neighborhood attacks," ICDE 2008, IEEE 24th International Conference on Data Engineering, pp. 506 -515, April 2008.

[10] Y. A. Kim and M. A. Ahmad, "Trust, distrust and lack of confidence of users in online social media-sharing communities," Knowledge-Based Systems, vol. 37, pp. 438-450, 2013.

[11] L. Ding, D. Steil, B. Dixon, A. Parrish, and D. Brown, "A relation context oriented approach to identify strong ties in social networks," Knowledge-Based Systems, vol. 24, no. 8, pp. 1187-1195, 2011.
[12] T. H.-J. Kim, L. Bauer, J. Newsome, A. Perrig, and J. Walker, "Access right assignment mechanisms for secure home networks," Journal of Communications and Networks, vol. 13, no. 2, pp. 175 -186, April 2011.

[13] B. Zhou and J. Pei, "Preserving privacy in social networks against neighborhood attacks," ICDE 2008. IEEE 24th International Conference on Data Engineering, pp. 506-515, April 2008.

[14] Y. A. Kim and M. A. Ahmad, "Trust, distrust and lack of confidence of users in online social media-sharing communities," Knowledge-Based Systems, vol. 37, pp. 438-450, 2013.

[15] T Fenner, M Levene, G Loizou, and GRoussos, "A stochastic evolutionary growth model for social networks," Computer Networks, vol. 51, no. 16, pp. 4586-4595, 2007

[16] B. Carminati, E. Ferrari, R. Heatherly, M.Kantarcioglu, and B.Thuraisingham, "Semantic web-based social network access control," Compute Fraud and amp Security, vol. 30, no. 23, pp. 108 - 115, 2011.

[17] C. M. Ma, Y. Zhuang, and S. Fong, "Information sharing over collaborative social networks using xacml," 2011 IEEE 8th International Conference on e-Business Engineering(ICEBE), pp. 161-167, Oct. 2011.

[18] M. Holanda, A. Queiroz, A. Araujo, and J. de Castro. Mobisn, "A framework for developing mobile social networks in manet," in Proc. 2012 7th Iberian Conference on Information Systems and Technologies (CISTI), 2012, pp. 1-5.

[19] A. Harrer and A. Schmidt, "Blockmodelling and role analysis in multi-relational networks," Social Network Analysis and Mining, pp. $1-19,2013$

[20] M. Takaffoli, F. Sangi, J. Fagnan, and O. R. Zane, "Community evolution mining in dynamic social networks," Dynamics of Social Networks 7th Conference on Applications of Social Network Analysis ASNA 2010, Procedia - Social and Behavioral Sciences, vol. 22, pp. 49-58, 2011.

[21] B. Bringmann, M. Berlingerio, F. Bonchi, and A. Gionis, "Learning and predicting the evolution of social networks," Intelligent Systems, IEEE, vol. 25, no. 4, pp. 26-35, 2010.

[22] N. P. Nguyen, T. N. Dinh, Y. Xuan, and M. T. Thai, "Adaptive algorithms for detecting community structure in dynamic social networks," INFOCOM, 2011 Proceedings IEEE, pp. 2282-2290, 2011.

[23] L. Zenk, C. Stadtfeld, and F. Windhager, "How to analyze dynamic network patterns of high performing teams," Procedia - Social and Behavioral Sciences, The $1^{\text {st }}$ Collaborative Innovation Networks Conference-COINs2009, vol. 2, no. 4, pp. 6418-6422, 2010.

[24] Z. Tian and N. Samaan, "A novel approach for modeling and managing spontaneous social communities over manets," Social Network Analysis and Mining, pp. 1-16, 2013.

[25] K. S. Cho, J. Y. Yoon, L. J. Kim, J. Y. Lim, S. K. Kim, and U.-M. Kim, "Mining information of anonymous user on a social network service," 2011 International Conference on Advances in Social Networks Analysis and Mining (ASONAM), pp. 450-453, 2011.

[26] N. Din and S. Haron, "Knowledge sharing as a culture among malaysian online social networking users," Procedia - Social and Behavioral Sciences, 16-18 July, vol. 50, pp. 1043-1050, 2012.

[27] A. Cucchiarelli, F. DAntonio, and P. Velardi, "Analyzing collaborations through content-based social networks," A. Abraham, A.-E. Hassanien, and V.v Snel, editors, Computational Social Network Analysis, Computer Communications and Networks, pp. 387-409, London: Springer, 2010.

[28] A. L. Barabsi, H. Jeong, Z. Nda, E. Ravasz, A. Schubert, and T. Vicsek, "Evolution of the social network of scientific collaborations," Physica A: Statistical Mechanics and its Applications, vol. 311, no. 34, pp. 590-614, 2002.

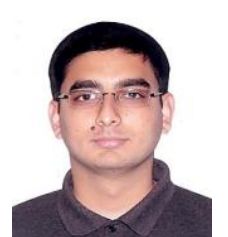

Swapnil Shantaram Ninawe received his BE in electronics engineering from Shri Ramdeobaba Kamla Nehru Engineering College, Nagpur, India, and ME Degree in Electrical Communication Engineering from Indian Institute of Science, Bangalore, India, in 2008 and 2011 respectively. Currently he is pursuing his Ph.D degree on Social Networks under the guidance of Prof. Pallapa Venkataram in the Department of Electrical Communication Engineering at Indian Institute of Science, Bangalore, India. His research interests are in the areas of Social Networks, Knowledge Based Systems and exploring evolution of actors in Social Networks. He is a student member of IEEE. 


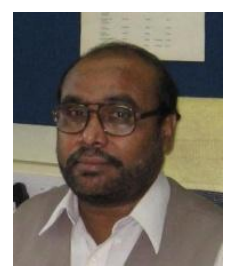

Pallapa received his $\mathrm{Ph} . \mathrm{D}$. Degree in information sciences from the University of Sheffield, England, in 1986. He is currently the chairman for center for continuing education, and also a Professor in the Department of Electrical Communication Engineering, Indian Institute of Science, Bangalore, India. Dr.

Pallapa's research interests are in the areas of Wireless Ubiquitous Networks, Social Networks, Communication Protocols, Computation Intelligence applications in Communication Networks and Multimedia Systems.

$\mathrm{He}$ is the holder of a Distinguished Visitor Diploma from the Orrego University, Trujillo, PERU. He has published over 150 papers in International/national Journals/conferences. Written three books: Mobile and wireless application security, Tata McGraw-Hill, Communication Protocol Engineering, Prentice-Hall of India (PHI), New Delhi, 2004 (Co-author: Sunil Manvi), and Multimedia: Concepts \& Communication, Darling Kinderley(India) Pvt. Ltd., licensees of Pearson Education in South Asia, 2006. Edited two books: Wireless Communications for Next Millennium, McGraw-Hill, 1998, and Mobile Wireless Networks \& Integrated Services, John Wiley \& Sons(Asia) Pvt. Ltd., 2006(Co-editors: L.M.Patnaik \& Sajal K. Das). Written chapters for two different books, and a guest editor to the IISc Journal for a special issue on Multimedia Wireless Networks. He has received best paper awards at GLOBECOM'93 and INM'95 and also CDIL (Communication Devices India Ltd) for a pape published in IETE Journal. He is a Fellow of IEE (England), Fellow of IETE(India) and a Senior member of IEEE Computer Society. 